\title{
La ciencia política en Panamá: UN NUEVO PUNTO DE PARTIDA
}

\author{
Salvador Sánchez González \\ Universidad Católica Santa María la Antigua, Panamá
}

\begin{abstract}
Resumen
Se ha dicho que no hay ciencia política en Panamá. Sin embargo, este artículo registra un panorama más diverso y complejo: ilustra cómo se establecen las Facultades de Derecho "y Ciencias Políticas", y cómo la precaria enseñanza de la disciplina queda atrapada entre juristas. Esto implica una preocupación excesiva por la organización constitucional del Estado. También expone el desarrollo de una vía paralela, con el ascenso de la sociología y otras ciencias sociales, que examinan la sociedad en su totalidad, y desde ángulos predominantemente históricos y económicos. Las investigaciones en ambas vertientes son numerosas y abordan una gran cantidad de temas durante los últimos 25 años. Sin embargo, el final simultáneo del régimen militar y de la guerra fría brinda oportunidades adicionales para la labor académica. Para la ciencia política panameña, podría tratarse de un nuevo y más sólido punto de partida.
\end{abstract}

\begin{abstract}
It has been said that there is no political science in Panama. However, in this article a more complex and rich landscape is registered: It shows how the Law "and political sciences" schools were established, and how the precarious teaching of political science became trapped between jurists. This implies an excessive preoccupation for the constitutional organization of the State. It is also exposed on this article, how a parallel way was develop, with the ascent of sociology and other social sciences, that examine the entire society, and from angles predominantly historic and economic. The researches on both basins are numerous and examine a great amount of topics during the last 25 years. However, the simultaneous end of the military rule and the cold war, brings additional opportunities for academic work. For Panamanian political science, it may be a new, and more solid, starting point.
\end{abstract}

\section{PALABRAS CLAVE • Ciencia Política • Panamá • Disciplina • Programas Académicos}

Es oportuno comenzar señalando que el presente artículo busca brindar un panorama de la ciencia política en la República de Panamá: dar una mirada a los últimos 25 años, y hacer un balance sobre las mayores deficiencias y fortalezas de la disciplina en el Istmo ${ }^{1}$. Es una tarea que se presentó en un principio como irrealizable, tomando en cuenta la aparente ausencia de una masa crítica de profesionales de la ciencia política y de estudios politológicos significativos que justificaran esta investigación. Signo evidente de esta situación es que no existe en las universidades panameñas licenciatura ni programa de postgrado en ciencia política. Sin embargo, este trabajo sirvió para contrastar esa percepción inicial sobre el estado actual de la ciencia política panameña, a través de una exploración más cuidadosa. No cuesta constatar que la falta de institucionalización adecuada de la disciplina no ha impedido su aparición. Un diagnóstico certero es por tanto fundamental, si se quiere emprender acciones para superar la precariedad de la disciplina en el Istmo. Y, si bien este artículo dejará seguramente preguntas sin respuesta, debe verse como un eslabón en la cadena de esfuerzos similares ensayados previamente. 


\section{CIENCIA POLÍTICA: EL CONCEPTO IMPERANTE SOBRE LA DISCIPLINA}

Para explicar la situación de la ciencia política panameña habría que precisar que Panamá destaca por haber sido el hogar de Justo Arosemena. Político y jurista, Arosemena produjo una obra de juventud titulada Apuntamientos para la Introducción a las Ciencias Morales y Políticas (Arosemena, 1840), mucho antes de su contribución intelectual más reconocida, sus Estudios Constitucionales (Arosemena, 1888). Estos y otros aportes a las ciencias sociales durante el siglo XIX, convierten a Arosemena en fuente de inspiración para muchos investigadores contemporáneos. Para efecto de esta monografía, sirve su mención para explicar la solera de la frase "ciencias políticas" entre los intelectuales panameños, influidos durante el siglo XIX por la obra de autores franceses, entre ellos Condorcet, Constant y Boutmy, este último fundador de la Escuela Libre de Ciencias Políticas (1872).

Al hablar de "ciencias políticas" se plantea el problema de la unidad o pluralidad de las ciencias sociales. La especialización en ciencias sociales, consecuencia del positivismo, fragmenta la realidad en áreas de conocimiento desconectadas unas de otras: la política, la economía, el derecho. La especialización haría perder de vista el bosque, por concentrar la atención en árboles aislados, y serviría para ocultar lo que para el marxismo es fundamental resaltar: la estructura de clases y los mecanismos de explotación que atraviesan la sociedad en su conjunto. De todas formas, los estudios de derecho y la profesionalización de la disciplina eran ya algo consolidado al escindirse Panamá de Colombia, en 1903. La creación de escuelas de derecho y ciencias políticas se inserta en ese contexto. La profesionalización de la ciencia política y la adquisición de autonomía académica estaban aún gestándose en todo el mundo. Sin embargo, la importancia que para el Estado tenían muchas de las distintas ciencias sociales no jurídicas era evidente. Los cursos universitarios correspondientes consistirían por tanto en la reunión de los conocimientos producidos por la economía, la historia, o la filosofía, relevantes para el conocimiento del Estado y la política.

En el prólogo a la quinta edición de sus Principios de Ciencia Política, Quintero señala que la licenciatura en derecho y ciencias políticas engloba estudios de derecho, y de otras ciencias sociales no jurídicas "que en una u otra forma se ocupan de algún aspecto esencial del Estado. Entre tales ciencias suelen incluirse la hacienda pública, la estadística, la economía política, la criminología y otras más" (Quintero, 1973: III).

Las Facultades de Derecho en Panamá receptaron el estudio de las ciencias políticas desde su fundación. Así ocurrió incluso en las escuelas de derecho y ciencias políticas que antecedieron a la creación de la Universidad de Panamá, aun y cuando no existiera un curso propiamente de ciencia política². Esto, porque lo que hace a los programas de ciencias políticas ha sido tradicionalmente la presencia de diversos cursos de ciencias sociales que tratan del Estado, y no la inclusión del solitario curso de Introducción a la ciencia política. Sin embargo, esto es precisamente lo que suele percibirse y criticarse, al suponerse la expedición de grados académicos en derecho y ciencias políticas como una impostura. En su aguda monografía sobre el tema, Rubén Darío Rodríguez Patiño plantea, sin embargo, que esos programas son cuestionados por las razones equivocadas. Al ser programas "de ciencias políticas", deben denominarse así. Cuestionar esos

2 El programa de estudios de la primera Escuela de Derecho y Ciencias Políticas incluía un curso de economía política, cuyos alcances desconocemos. 
programas es admisible, pero sería incorrecto afirmar que pretenden engañar, en la medida que son precisamente lo que dicen ser (Rodríguez Patiño, 2004)3.

\section{LA ENSEÑANZA DE "PRINCIPIOS DE CIENCIAS POLÍTICAS"}

En 1935 se crea la Universidad de Panamá. La introducción de la ciencia política en la Universidad de Panamá fue obra de José D. Moscote, su primer Decano General (Quintero, 1966: I). Al finalizar la Segunda Guerra Mundial, el área de ciencia política es integrada por dos materias: la Introducción a la ciencia política y los principios de la democracia, que desapareció pronto (Figueroa Navarro, 1983: XXXI). Quintero gana la cátedra de ciencia política en 1944, y se mantiene en ella durante las décadas siguientes.

El escenario universitario actual es diferente. La enseñanza se ha expandido, disparándose el número de centros de enseñanza superior que ofrecen licenciaturas en derecho y ciencias políticas ${ }^{4}$. Sin embargo, la pluralidad no ha venido de la mano de la diversidad. El modelo de la Facultad de Derecho de la Universidad de Panamá se ha reproducido a lo largo del país, con todas sus deficiencias.

Los docentes de ciencia política, por ejemplo, son en la actualidad un grupo numeroso, entre los que predominan los de exclusiva formación jurídica ${ }^{5}$. Dice al respecto la profesora De Sanctis, que

(...) hay pocos politólogos panameños graduados como tales, de los cuales ninguno es docente en la Universidad de Panamá, mientras que la mayoría de los profesores que conforman el Departamento de Ciencia Política son abogados que ejercen su profesión, casos en los que el estudio de esta ciencia es claramente la actividad secundaria (...) (De Sanctis; en Rodríguez Patiño, 2005: 3-4).

Como se ha indicado, se trata de una situación generalizada en la universidad panameña, aunque fue en la Universidad de Panamá donde se debatió la exigencia de título en derecho a los profesores que enseñaban ciencia política (con la paradójica exclusión de los académicos con titulación exclusiva en la disciplina), a mediados de los años 90. El conflicto resultante llevó a mejorar levemente la conciencia pública sobre la situación marginal de la disciplina, pero la exclusión de

3 En este punto, por lo menos, es obvio que la perspectiva de las ciencias políticas no se lleva hasta las últimas consecuencias en las universidades panameñas. Admitiendo que se incorporan a la licenciatura en derecho y ciencias políticas contenidos de las distintas ciencias sociales, estos contenidos deben ser presentados por especialistas: si se trata de principios de economía, por economistas. Si se trata de principios de sociología, por sociólogos. Y sin embargo, no son frecuentes los politólogos a cargo de los cursos de principios de ciencia política. Una causa probable de esta situación es la relativa escasez de egresados de ciencia política, y la demanda relativamente alta de profesores para los cursos de principios de ciencia política que se dictan en las Facultades de Derecho.

La primera universidad privada fue la Universidad Católica Santa María La Antigua (1965). A ella se agregaron desde finales de los 80 y en rápida sucesión, muchos otros centros de enseñanza superior: Universidad del Istmo (1987), Universidad Latina de Panamá (1991), Universidad Latinoamericana de Ciencia y Tecnología (1991). Tanto la Universidad de Panamá como las universidades privadas tienen sedes en la ciudad de Panamá, y en las principales cabeceras provinciales, en las que también se dictan cursos de principios de ciencia política. Además, una nueva universidad estatal, la Universidad Autónoma de Chiriquí, fue creada en 1995. Todos estos centros de estudios ofrecen licenciaturas en derecho y ciencias políticas.

5 Enseñan en la Universidad de Panamá Isidro Acosta, Miguel A. Bernal, Freddy Blanco, Aleyda Giulia De Sanctis, Raúl González, Carmelo González, Eddy Hernández, Svetlana Jaramillo, Julio Miller, Guillermo Moreno, Rubén Darío Rodríguez Patiño, Daniel Vargas. En la Universidad Latina enseñan Carlos de Diego, Allys de la Rosa y Dennis Allen. En la Universidad Católica Santa María La Antigua, han enseñado Francisco Vega, Dennis Allen, Enrique Noel, Jorge Hernán Rubio y Salvador Sánchez. En la Columbus University, dicta Luis De León Arias. 
hecho persiste, lo que consolida en las aulas la perspectiva institucionalista tradicional, centrada en el estudio del Estado desde el derecho, que también ha sido la norma en los programas de estudio. La creación de un Departamento de Ciencia Política en la Facultad de Derecho y Ciencias Políticas (1996), por ejemplo, no ha significado mayor cambio pese a que bajo él se organizan ahora los cursos de ciencia política y los de derecho constitucional.

Por otro lado, el hito indiscutible en los estudios superiores vinculados con la ciencia política, lo es la maestría en estudios políticos, que por poco tiempo (hubo una única promoción) se dictó en la Facultad de Derecho y Ciencias Políticas de la Universidad de Panamá, entre 1994-95, bajo la coordinación de Jorge Giannareas ${ }^{6}$. Como lo indica la propia denominación del programa, le caracterizaba una aproximación interdisciplinaria al fenómeno político.

Un hito también, aunque de signo contrario, es la implantación general en la universidad panameña de formas de alcanzar la licenciatura sin realizar el tradicional trabajo de graduación (proceso que corresponde al inicio de la presente década). El conjunto de los trabajos de graduación constituía parte importante de la poca investigación en ciencia política que se ensayaba en las Facultades de Derecho y Ciencias Políticas de todo el país.

También es destacable que durante décadas hayan funcionado en Panamá algunas universidades estadounidenses, entre ellas, el ahora extinto Panama Canal College y la Florida State University, cobijadas ambas en la Zona del Canal de Panamá. Desaparecido el enclave, la Florida State University se ha instalado formalmente bajo la jurisdicción nacional desde 1999, y suele tener programas de licenciatura en los que se integraban cursos que vale la pena mencionar ${ }^{8}$. Más recientemente, la Louisville University, ha estrenado un programa de maestría en relaciones internacionales, donde se dictan también cursos de ciencia política ${ }^{9}$. Estas universidades abordan la ciencia política como es entendida en la academia estadounidense, y pueden llegar a tener un impacto significativo por su vinculación internacional, y por emplear a algunos politólogos panameños formados en universidades anglosajonas.

\section{CIENCIA POLÍTICA: LOS PROGRAMAS}

Las reflexiones sistemáticas sobre lo social, hechas en Panamá o por panameños a principios del siglo XX, fueron institucionalistas. Los fenómenos políticos se examinaban dando prioridad al conocimiento del Estado a través de los textos constitucionales. Esto era coherente con el estado de la disciplina, porque a finales del siglo XIX y principios del siglo XX el análisis de lo político estaba limitado a las investigaciones morales, al relato histórico sobre acontecimientos políticos, y a los estudios jurídicos sobre el Estado. Estas aproximaciones servían para que la primera ciencia política

6 Cursaron ese programa: Raúl Leis, Rubén Darío Rodríguez Patiño, Enrique Noel, Giulia De Sanctis, Olmedo Beluche y Enoch Adames, entre otros.

7 La conferencia inaugural de la maestría, dictada por Simeón González el 6 de enero de 1995, fue luego publicada. En ella se propuso un agrupamiento temático de la ciencia política en torno a estructuras políticas, procesos politicos y conducta política (González H., 1995:10).

8 Entre los cursos están: Studies in International Politics: Latinamerica, Introduction to Comparative Government and Politics, y Politics of Latinamerica, dictadas por Matthew Atlee (M.A. en Political Science de York University), e Introduction to Political Thought, dictada por Carlos Guevara Mann (PhD. Political Science, University of Notre Dame).

9 Entre los cursos están: Scope of Political Science, dictada por Alfredo Castillero Hoyos (PhD. Political Science, Portsmouth). Otros cursos son Methods of Political Research, y Comparative Politics. 
no pudiera distinguirse del análisis jurídico formal, de la interpretación y comparación del derecho positivo, y de la lectura más o menos actualizada de los clásicos del pensamiento político.

Sin embargo, a mediados del siglo XX se consolidan los métodos de la psicología y la sociología aplicados al análisis de los fenómenos políticos, y se hace posible la denominada revolución conductista, que introduce los métodos del sondeo de opinión, la entrevista, y una variedad de nuevas técnicas estadísticas útiles para cuantificar el comportamiento de los actores políticos. En Panamá, los cursos universitarios no fueron impactados por estos cambios, pese a que el propio autor de Principios de Ciencia Política, era conciente de ellos. Quintero señaló en la "Introducción" de su manual:

Cabe advertir que en este texto nos ceñiremos al programa vigente de la materia en la Universidad de Panamá y que seguiremos, por ello, los planteamientos tradicionales de la ciencia política. Nos limitaremos, pues, a describir con palabras y apreciaciones nuestras, los objetivos e indagaciones de la ciencia política clásica. Tenemos, sin embargo, que objetar a este tipo de ciencia política su preocupación esencial por formalismos jurídicos antes que por las causas profundas de los fenómenos políticos. Creemos que la ciencia política del futuro habrá de ocuparse preferentemente en la búsqueda de estas causas y acercarse y vincularse cada vez más a la economía, la sociología, la psicología y la ética (Quintero, 1973: 9-10).

Sin embargo, el plan de estudios de ciencia política en la Universidad de Panamá continuó por la senda tradicional, durante décadas ${ }^{10}$.

Mientras tanto, la ciencia política tradicional sufrió una crítica sistemática fuera de nuestras fronteras. Se señaló que los estudios politológicos se dedicaban a pocas áreas geográficas, describían solamente los sistemas políticos sin preocuparse de hacer elaboraciones teóricas y daban una excesiva atención a las instituciones y a las normas. El producto de las investigaciones realizadas con esta orientación exhibía frecuentemente una falta de integración del estudio puramente exegético de los textos constitucionales, y los modelos de comportamiento político. Sin embargo, esa disociación es básicamente la tendencia que ha persistido a todo lo largo del siglo, en la comprensión dada en Panamá a una ciencia política vinculada al manual hegemónico en la enseñanza universitaria de la ciencia política en las Facultades de Derecho y Ciencias Políticas.

Sin embargo, los programas de estudio han sido recientemente renovados en la Universidad de Panamá. El área de ciencia política en la carrera de Derecho y Ciencias Políticas incluye dos cursos clásicos, uno obligatorio denominado principios de ciencia política, y otro optativo, llamado historia de las ideas políticas. Actualmente, la asignatura principios de ciencia política tiene como finalidad

que el estudiante pueda acceder al discernimiento científico del Estado y, en general, de la política; de manera que estén a su disposición los instrumentos teóricos y metodológicos para comprender el acontecer político nacional e internacional. En particular, el cursante deberá ser motivado a estudiar las principales interpretaciones acerca del origen y naturaleza del poder político, al igual que a razonar en torno a la compleja problemática sociopolítica de los tiempos que corren (Rodríguez Patiño, 2005: 9).

10 Al darse la quinta edición de su obra, en 1966, Quintero agregó un prólogo dedicado en gran parte a tomar nota de los múltiples cambios que sufría la disciplina, pero siguió justificando la conservación del texto original de su manual dado que seguía adecuándose al programa de estudios de la Facultad de Derecho y Ciencias Políticas de la Universidad de Panamá, y a que se ajustaba también a la lista tipo de áreas temáticas presentada por UNESCO en 1948, lista que fijaba las fronteras de la ciencia política. 
Adicionalmente se señala que la materia se estructura en torno a cinco ejes: teoría política, métodos de la ciencia política, economía, política y sociedad, instituciones políticas e ideologías políticas.

Los programas de estudio en la Universidad Católica Santa Maria La Antigua también están siendo objeto de revisión, pero actualmente se describe la materia de ciencia política como

(...) el estudio del Estado, y sus componentes. Estudia el gobierno, que pudiera llamarse el "motor" del Estado, y la relación existente entre el gobierno y sus gobernados. En el primer semestre del curso se estudia primordialmente el tema del Estado y del Gobierno. Se estudia el origen del Estado, las diferentes clases de Estados, y las diferentes teorías a través de la historia sobre los fines ideológicos del Estado. Se destaca también el lugar del gobierno dentro de la maquinaria estatal, sus funciones, y las diferentes formas de gobierno según los maestros clásicos de la teoría política ${ }^{11}$.

Durante el segundo semestre del curso se inicia:

(...) el estudio básico del constitucionalismo y la constitución, se destaca la Constitución Panameña, como también los principios fundamentales de las relacionales internacionales y política internacional, limitaciones del poder nacional, problemas y soluciones en relación con la paz en el siglo XX. Se finaliza el segundo semestre con el estudio de la opinión pública, su dinámica y su importancia en la formación de la opinión política ${ }^{12}$.

De la lectura de los programas se puede concluir que a pesar que los cursos de principios de ciencia política están firmemente anclados en el institucionalismo tradicional, los planes de estudio en las dos principales facultades de Derecho y Ciencias Políticas están evolucionando, dando más oportunidades para dar cabida a una ciencia política auténtica, abarcadora del estudio del Estado y de lo institucional en general, pero no solamente de lo institucional. Los cambios no podrán ser sustanciales, sin embargo, si por lo menos no se disminuye el énfasis jurídico de la formación de los docentes responsables de desarrollar esos programas en clase, y se le reemplaza por un énfasis politológico. Así, aunque se incluya el estudio de la opinión pública entre los contenidos de los cursos de la disciplina, el abordaje científico de las encuestas, por ejemplo, no podrá hacerse sin la debida calificación del cuerpo docente.

La atención que despertó la revolución conductista sirve para retomar lo que ya Gandásegui ha señalado: que a nivel de teorías y escuelas en ciencias sociales, sobresalen tan sólo el funcionalismo, introducido a Panamá por Hernán Porras, y el marxismo, desarrollado principalmente en torno a la figura de Soler (Gandásegui, 1998). Así, quienes absorbieron la influencia conductista en las ciencias sociales han sido, por razones comprensibles, los psicólogos y los especialistas en mercadeo, aunque no los pocos politólogos de academia ${ }^{13}$. Es por esto que la crisis de las investigaciones conductistas no ha tenido mayor consecuencia para la ciencia política en Panamá. Igual cabe decir de la escuela de la elección racional, hegemónica en tantos otros lugares. Así, tampoco puede atribuírsele al predominio de estas corrientes de la ciencia política la ausencia de contribuciones significativas a la teoría política, la mayor parte de las cuales se circunscribe al entorno intelectual del marxismo local (Soler, 1963; Adames, 1996; 2002).

http://www.usma.ac.pa/licenciaturas/fac_derecho/lic_Der_Cienc_Pol_descrip.html

lbidem.

Dichter \& Neira Marketing Consultants fue fundada en 1987 y es desde entonces la más emblemática empresa de estudios de mercadeo y opinión pública. 
Curiosamente, la aparición del neo-institucionalismo en ciencia política parece dar una segunda oportunidad para la transformación de la escena académica. En tiempos del primer institucionalismo, por ejemplo, el análisis de las elecciones no podía ser científico, ni aun proponiéndoselo. La disponibilidad contemporánea de datos creíbles respecto a los torneos electorales desde 1991, ha permitido profundizar en esos estudios. Habría que decir que puede verse reflejado en los temas examinados por los nuevos investigadores (Pérez, 1993; Franco R., 2001; Brown, 2002).

\section{TEMAS DE LA CIENCIA POLÍTICA EN PANAMÁ}

Sobre la evolución de las ciencias sociales en Panamá, son de gran utilidad los estudios de Alfredo Figueroa Navarro. Sus múltiples trabajos expresan en parte la situación de la ciencia política, incluyendo los aportes bibliográficos realizados hasta el momento.

En su antología sobre el desarrollo de las ciencias sociales en Panamá durante los primeros 80 años de la República, Figueroa Navarro recoge, como ilustración de la ciencia política panameña, cinco ensayos publicados entre 1922 y 1978 (Figueroa Navarro, 1983). La selección tiene la virtud de recoger escritos de autores que efectivamente estudiaron ciencia política: Quintero en Estados Unidos (Georgetown, Estados Unidos); Renato Pereira y Vilma Ritter (Universidad de París, Francia). Los temas de los ensayos recogidos incluyen el estado de la ciencia política (Quintero) y el papel de las fuerzas armadas en América Latina y Panamá (Pereira, 1978; Ritter, 1983).

Junto a estos ensayos, Figueroa Navarro incluyó una bibliografía sobre las distintas ciencias sociales, en la que lista treinta publicaciones de ciencia política, de 24 autores diferentes, incluyendo tres no panameños, lo que sugiere la necesitad de hacer un estudio adicional sobre Panamá en los estudios politológicos extranjeros ${ }^{14}$. Entre las obras listadas como producciones de la ciencia política panameña, predominan las que hacen de los partidos políticos su objeto, pero se encuentran perfilados también otros temas: el debate que se dio en el seno del liberalismo panameño a principios del siglo XX, y el examen al rol de los militares en la política, particularmente después del establecimiento del régimen militar en Panamá, en 1968.

En una obra posterior de Figueroa Navarro, dedicada a examinar la evolución de las ciencias sociales durante la década inmediatamente siguiente a su estudio de 1983, el autor no identifica aportes adicionales en el campo de la ciencia política, como sí hace, por ejemplo, con el apartado que dedica a la sociología y a otras ciencias sociales (Figueroa Navarro, 1993:107-136). Llama la atención esta omisión, porque se trata de un período de gran actividad intelectual y política, que se corresponde con la etapa final del régimen militar, la invasión estadounidense y el establecimiento de la institucionalidad democrática.

En 1988 salió a la luz una antología de pensamiento político panameño, correspondiente al siglo XIX y XX (Soler, 1988). Aunque fue terminada en 1982, el tomo vio la luz con mucho retraso, lo que explica la ausencia de materiales adicionales correspondientes a esa década. Sin embargo, es una de las colecciones más ricas e interesantes de actas, artículos y discursos políticos panameños.

14 Reconociendo que ha habido desde hace décadas estudios politológicos sobre Panamá en la academia estadounidense (Pippin, 1964; Ropp, 1982; Scranton, 1991; Conniff, 1992; Millet, 1996; Sánchez, 2000), y más recientemente, estudios desarrollados por politólogos españoles (García Díez, 2003; Otero, 2004). 
Otra bibliografía reciente, incluye un listado de obras de ciencia política publicadas durante los primeros 100 años de República. Se incluyen 22 publicaciones, de 19 autores distintos, todos panameños (Fábrega y Soler, 2003). Una excelente iniciativa, que debe ser valorada como un primer acercamiento a una bibliografía realmente exhaustiva, todavía pendiente. Lamentablemente, también se echa de menos un criterio homogéneo a la hora de clasificar las obras que fueron finalmente incluidas. Ambas circunstancias resultan evidentes en el listado referente a ciencia política, cuya presencia en una bibliografía jurídica de por sí resulta desconcertante. Entre las obras incluidas en esta bibliografía y que fueron publicadas después de 1980 hay varias que revisten interés. Pueden destacarse el análisis del primer cambio constitucional en la Constitución del régimen militar (Arroyo, 1980) y unas reflexiones sobre historia de las ideas políticas en Panamá (Pereira Burgos, 1986).

Debe señalarse que entre las publicaciones del período destacan varias que tienen a la propia ciencia política como objeto de estudio, y particularmente la disciplina en Panamá. Así, un conjunto de monografías publicadas por Simeón González (González H., 1995; 1983) y Rodríguez Patiño (Rodríguez Patiño, 2005; 2004; 1998ª) han preparado el terreno para una toma de decisión conciente sobre la conveniencia y viabilidad de establecer programas universitarios en ciencia política, y sobre la forma que esos programas deben adoptar.

Por otro lado, hay algunos problemas que han despertado profundo interés en los investigadores panameños. Indudablemente, uno de esos temas es el de la invasión a Panamá por los Estados Unidos, sobre el cual, entre publicaciones panameñas y extranjeras, hay varios centenares de libros y artículos. La Revista Tareas, por poner sólo un ejemplo, dedicó los números 74 (1990), 77 (1991) y 81 (1992), a discutir la invasión de 1989.

Durante el régimen militar (1968-89), y posteriormente, se reflexionó sobre su naturaleza y sucesivas transformaciones. Comprensiblemente, las posiciones son muy numerosas, y abundan los matices entre los distintos autores, unos receptivos respecto del reformismo militar (Soler, 1975; Cortés, 1986; Soler Torrijos, 1988), y otros críticos de sus rasgos autoritarios (Pereira Burgos, 1991; Guevara Mann, 1996; Janson Pérez, 1998). La dinámica de la transición democrática también ha sido objeto de algunos estudios (Castro, 1991; Janson, 1993; Sánchez González, 2004), lo mismo que su efecto más notorio, la desmilitarización del país (Arias Calderón, 2001).

Los tópicos relacionados con la democracia se establecerán posteriormente (Gandásegui, 1989; Franco, 1993; Castillero Hoyos, 1996; Noel, 1999), y cada vez con más frecuencia, el tema de los partidos políticos y las elecciones (Pérez, 1995; Adames, 1998; Brown, 2002; Sánchez González, 2004).

Con ocasión de la edición No. 100 de Tareas, se examinaron los artículos publicados (Figueroa Navarro, 1999) ${ }^{15}$. Los temas más consistentemente tratados por la revista son los relacionados con la crisis

15 El listado de temas de ciencia política abordados en los cien primeros números de la Revista Tareas son, según Figueroa Navarro: "los problemas canaleros, situación del proletariado, reforma universitaria, problemas nacionales, Tribunal Russell, comentarios a los proyectos de tratados de 1967 con los Estados Unidos de América, función de los partidos políticos, problemas de la violencia y de la justicia política, populismo militar, bonapartismo, desarrollo político, no alineación, tercermundismo, política universitaria, liberación nacional, crisis del Estado, reforma agraria, autoritarismo, feminismo, pensamiento político, marxismo, deuda externa, teología de la liberación, anarquismo, negritud, movimientos populares, gobernabilidad, trabajo invisible de la mujer, nuevo orden mundial, indignidad, sector informal, contaminación ambiental, multiculturalidad, desarrollo sustentable, postmodernidad, pobreza". Sin embargo, ni la clasificación de los artículos en históricos, económicos, sociológicos y políticos, ni el listado de los temas supuestamente políticos, resultan convincentes. Vale la lista como muestra de la diversidad de asuntos tratados (Figueroa Navarro, 1999: 127-136). 
económica que sacudía a toda la región en la década de 1980 (Pinzón, 1988), y los análisis de coyuntura dedicados a los diferentes momentos que atravesó el régimen militar hasta su desaparición como consecuencia de la invasión estadounidense de diciembre de 1989 y la ocupación militar (Camargo, 1992), sin abandonar los debates que se llevan a cabo en el resto del mundo, como el fin de la historia (Ungo, 1993), la globalización (García, 2005), y la gobernabilidad (Adames, 2000).

Al acercarse el centenario de la República, la evaluación del pasado histórico se hizo obligado, y así vemos cómo se examinan períodos y episodios que habían sido relativamente desatendidos, tales como el lapso entre la independencia de España y el establecimiento del Estado del Istmo en 1841 (Lasso, 2004) o los inestables primeros años del siglo XX (Arias Calderón, 2004; McKay, 2004). Sin embargo, las contribuciones más significativas trataron con la naturaleza del movimiento que dio nacimiento a la República en 1903, tema que generó un intenso y público debate. Las contribuciones a este debate pueden ser calificadas como representativas de la "leyenda dorada" (Arango, 1930; Ortega Brandao, 1931), de la "leyenda negra" (Terán, 1935; Díaz, 2003; Beluche, 2003), y de la "versión ecléctica" sobre la independencia de Panamá (Escarreola, 2002; Aparicio, 2003; Ricord 2003), como en su momento las agrupara Gasteazoro.

\section{DEBILIDADES Y FORTALEZAS DE LA CIENCIA POLÍTICA EN PANAMÁ}

Las debilidades de la ciencia política panameña no son pocas. En un escueto párrafo dedicado a la disciplina, Figueroa Navarro alcanza a señalar la necesidad de establecer una "licenciatura de politología" y un "Instituto de Estudios Políticos". Identifica ya en ese momento la existencia de politólogos "desvinculados de la Universidad" y el fenómeno que representa el hecho que "las mejores encuestas politológicas emanen de las distintas empresas de opinión pública que existen fuera de la Universidad" (Figueroa Navarro, 1993: 11).

La primera gran debilidad, señalada desde el inicio de este artículo, es la falta de un programa universitario de ciencia política. En parte se trata de un círculo vicioso: no hay suficientes politólogos porque no hay programas de formación en las universidades panameñas, y no hay programas de formación porque falta un numeroso e influyente grupo de politólogos que puedan apoyar su establecimiento. Como lo ha dicho Adames, el anidamiento en la universidad de unas disciplinas y no de otras, se deriva "de intentos desigualmente exitosos de quienes como intelectuales tienen acceso a determinadas dotaciones de poder que les permite incidir en la estructuración social, persuadir a otros actores y comunicar cómo difundir exitosamente determinadas concepciones de acción y relaciones sociales" (Adames, 2002). La falta de un programa universitario de ciencia política sería así menos un problema de la disciplina en tanto ciencia, que un problema de falta de poder de los politólogos, en los niveles de toma de decisión, en los centros de enseñanza universitaria panameños. Además, profundizar en el proceso de especialización en ciencias sociales pareciera ir contra el criterio de un sector importante de cientistas sociales, quienes incluso auguran el fin de la gran ruptura con las ciencias "duras"16.

16 Adames señala que quizá el "reto para las ciencias sociales panameñas pasa transitoriamente por una Facultad de ciencias sociales; pero lo que no puede subsistir son los territorios configurados más como espacios corporativos que académicos, y que con sentido de propiedad y feudo legitiman en nuestro medio la acriticidad, la abulia, y la inercia académica" (Adames, 2002). Se trata de la conclusión del único comentario que he identificado en Panamá, sobre el Informe Gulbekian, sobre el estado y el futuro de las Ciencias Sociales en el mundo. 
Estos asuntos son todos dignos de ser considerados, por lo que parece correcta la apreciación de Rodríguez Patiño cuando advierte que las confusiones en torno al concepto de ciencia política pueden amenazar el resultado de las iniciativas mejor intencionadas (Rodríguez Patiño, 2004). Esto, porque el concepto orienta el diseño del programa, y un programa mal diseñado, entiéndase, respecto del contexto panameño y nuestras específicas necesidades, fracasaría indudablemente.

Parte del problema, por lo tanto, radica en establecer cuáles son las necesidades que un programa de ciencia política podría satisfacer. Entre otras cosas, habría que determinar si el mercado de trabajo requiere de un número mayor de egresados de la disciplina que el que actualmente llega a Panamá tras estudiar en el extranjero en programas de licenciatura, maestría o doctorado en ciencia política. En principio parece que sí, básicamente porque en la actualidad parte significativa del trabajo de los politólogos en cuanto profesionales, es realizado por no especialistas, dentro y fuera de las universidades. Sin embargo, lo indispensable es crear y difundir conocimiento político, independientemente de si se alcanza ese objetivo mediante el establecimiento de licenciaturas 0 estudios de postgrado de ciencia política en Panamá17. Entre las alternativas está la creación de un auténtico centro de investigación político, para la creación de conocimiento, y la inversión estatal en becas para jóvenes panameños que se dispongan a estudiar la disciplina en universidades extranjeras.

En lo que atañe a este último punto, debe reconocerse que en la última década se han hecho serios esfuerzos estatales para promover la investigación, a través de la creación en 1992 de la Secretaría Nacional de Ciencia, Tecnología e Innovación (SENACYT) ${ }^{18}$, la cual ha impulsado distintos programas que podrían brindar oportunidades de aprovechamiento. Un objetivo de mediano plazo sería lograr que se le dé atención y prioridad a la ciencia política en el Plan Nacional de Ciencia, Tecnología e Innovación, y en los programas que ejecuta SENACYT. Los programas ejecutados en la actualidad, no dan prioridad a las ciencias sociales. El programa de becas en 2003 , por ejemplo, incluyó a 30 investigadores, pero las ciencias sociales no estaban incluidas en la convocatoria. En el programa 2005-2010 sí se incluyeron en la convocatoria las ciencias sociales, pero se otorgaron tan solo 21 becas entre doctorales (para formación de investigadores) y post doctorales (de especialización mediante entrenamiento) en todo el Programa Nacional de Investigación. Otros programas, como el de Fomento de Investigación y Desarrollo, han respaldado proyectos panameños de investigación científica por primera vez, con aportes de hasta U.S.D. 50.000.00 por proyecto. El programa también abarca las ciencias sociales, pero entre los 18 proyectos beneficiados sólo dos fueron de ciencias sociales ${ }^{19}$.

Esto nos lleva a la segunda gran debilidad de la ciencia política en Panamá: la ausencia de un centro de investigación o de un instituto de estudios dedicados específicamente a lo político. Debe decirse que en los años 90 se fundan varias organizaciones no gubernamentales que se

El establecimiento de esos programas no asegura ni la creación de conocimiento ni la incidencia directa en el desempeño de las instituciones que supuestamente deben impulsar. Esto se observa en algunas áreas de interés politológico, tales como la administración pública y las relaciones internacionales, que teniendo en pie programas de enseñanza universitaria, no han tenido el impacto previsto, sobre la calidad de la alta administración del Estado y sobre la gestión de la política exterior panameña, respectivamente.

18 http://www.senacyt.gob.pa/

19 Uno sobre la formación de la mentalidad urbana entre las clases de la ciudad de Panamá, entre 1930 y 1970 (Damaris Díaz, Miriam Miranda y Patricia del Cid) y otro que desde la Arqueología trata sobre la distribución y delimitación de las entidades político-culturales del gran Coclé pre-colombino (Julio Mayo). 
aproximan al conocimiento científico de la política: el Instituto de Estudios Políticos e Internacionales (IEPI)20, a inicios de la década, y el Centro de Iniciativas Democráticas (CIDEM) ${ }^{21}$, desde 1998. Ambas organizaciones promueven la publicación de investigaciones en temas políticos y constitucionales, organizan conferencias y realizan otras actividades de difusión. Sin embargo, ninguna es propiamente un centro de investigaciones politológicas. Los centros de investigación no estatales dedicados a las ciencias sociales, son fundamentalmente los muy consolidados Centro de Estudios Latinoamericanos (CELA) y el Centro de Acción Social de Panamá (CEASPA), cuyas figuras emblemáticas son Marco Gandásegui y Raúl Leis, respectivamente. También en este respecto la sociología es claramente predominante.

Una tercera debilidad resulta de la ausencia de nuevos manuales de ciencia política, que aspiren a reemplazar el texto de Quintero. Entre las contribuciones en esta área, se puede identificar la del abogado y ex Legislador Oydén Ortega Durán, quien publicó un breve manual de instituciones políticas (Ortega Durán, 1989). Más recientemente Enriqueta Davis Villalba y Freddy Blanco Muñoz han publicado un manual en dos tomos, sobre ciencia política y sociología política (Davis et al., 2001). Empero, está pendiente la preparación de una obra de mayores pretensiones. Es posible que los cambios de los programas de estudio contribuyan en el futuro próximo a preparar esos textos que tanta falta hacen.

En el mismo orden de ideas, se ha afirmado que no hay publicaciones periódicas que den cabida a la ciencia política en Panamá, en sentido exclusivo o especializado. Debe destacarse, sin embargo, la Revista Tareas, fundada en 1960, y que publica el CELA ${ }^{22}$. Dedicada a las ciencias sociales, con un marcado énfasis en las perspectivas históricas, sociológicas, y económicas, el examen de sus textos permite en gran medida contemplar la evolución de los temas de interés para los politólogos locales.

Por otro lado, una buena noticia en el período bajo examen ha sido la aparición de algunas editoriales preocupadas por los asuntos políticos, tales como Editorial La Prensa (Janson Pérez, 1993; Guevara Mann, 1994), y la Editorial Portobelo (Bernal, 1998; González H., 1995) que ha publicado muchas de las monografías panameñas de ciencia política escritas en los últimos años, en formatos muy económicos y accesibles.

Por otro lado, si la crítica al actual estado de la ciencia política se ocupa de constatar su fragilidad, ayuna como está de un programa de estudios universitarios, y de un centro de investigación

20 El IEPI, animado por su fundador, Miguel Antonio Bernal (Burdeos, Francia), ha publicado algunas obras de importancia (Janson Pérez, 1994, 1997; Guevara Mann, 1999; Bernal, 2000). También publicó en 1997 el número único de la Revista Estudios Políticos, que incluyó artículos de ciencia política, derecho constitucional, historia, y antropología.

21 El CIDEM está integrado a varias organizaciones internacionales de Ciencia Política (entre ellas, la Asociación Latinoamericana de Ciencia Política, ALACIP). EI CIDEM tiene una Comisión Permanente de Ciencia Política, y organizó durante 2004 el ler Encuentro Nacional de Politólogos.

22 En los últimos 25 años deben mencionarse también la Revista Panameña de Sociología, fundada por Gerardo Maloney (primer número: 1986; décimo número: 1999), y los Cuadernos Nacionales, del Instituto de Estudios Nacionales de la Universidad de Panamá. La Revista Parlamentaria Debate, publicada desde 2001 por la Asamblea Nacional, y dirigida por Harley James Mitchell Dale, también destaca. Otras publicaciones, como lustitia et Pulchritudo, de la Facultad de Derecho y Ciencias Políticas de la USMA, y el Anuario de la Facultad de Derecho y Ciencias Políticas de la Universidad de Panamá, incluyen ocasionalmente artículos de ciencia política. Ver por ejemplo: (Pedreschi, 1986; Ricord, 1990; Rodríguez Patiño, 1997). Por otro lado, las contribuciones sobre política nacional recogidas en la clásica Revista Cultural Lotería, que se ha publicado desde 1941, constituyen un acervo de inestimable valor. 
equiparable a los que actualmente defienden otras perspectivas de las ciencias sociales, no menos cierto es que los egresados de programas de ciencia política del extranjero encuentran su sustento dentro y fuera de las universidades panameñas. Los egresados de otras ciencias sociales, en general, han encontrado en su relación con el Estado y con las organizaciones internacionales establecidas en el país (PNUD, UNICEF, OIT, OEA, etc.) las oportunidades para su realización profesional. Se trata del mismo campo laboral al que concurren los titulados de ciencia política en el extranjero, y es el campo al que aspirarían acceder los egresados de un programa de ciencia política nacional, en caso de existir.

Sin embargo, hay que admitir que los politólogos han ido encontrando nichos, que si bien no son exclusivos, van perfilando el sentido práctico de la disciplina en escenarios diversos. Parte de esta transformación ha sido posible por el impacto del financiamiento público a los partidos políticos, que ha estimulado la capacitación y la preparación de las estructuras partidarias frente a los retos cotidianos y electorales. Aquellos politólogos que se decantan por el mercadeo político han encontrado en la modernización de las campañas electorales una oportunidad de brindar servicios relacionados con la dirección de las campañas propiamente dichas, la preparación, realización e interpretación de sondeos electorales y encuestas, y por esa vía, en la participación en los medios de comunicación de masas, como analistas políticos, consultores puntuales o empleados a tiempo completo.

\section{BALANCE FINAL}

Se observa que la ciencia política en Panamá, en los últimos 25 años, ha mantenido su primitiva veta institucionalista, conservándose los estudios formales dentro de las Facultades de Derecho y Ciencias Políticas, y sin programa alguno de licenciatura que promueva localmente el fortalecimiento de la disciplina a través de la generación de profesionales locales. En esta corriente, los temas principales continúan siendo los propios del derecho público, con particular relevancia aquellos del derecho constitucional, extendiéndose hasta el diseño de las instituciones y la ingeniería constitucional, con particular incidencia en el tratamiento de fenómenos tales como el parlamento, los partidos políticos y el sistema electoral, pero con predominio de la perspectiva jurídica.

Sin embargo, ya desde la aparición de la Revista Tareas, aprovechando la entrada al país de un número cada vez mayor de egresados de estudios de postgrado en ciencias sociales, y también de licenciados, graduados en centros universitarios extranjeros, particularmente latinoamericanos pero también europeos continentales, se introdujo el estudio sistemático de los hechos sociales a partir de nuevas metodologías. Si por un lado estas nuevas figuras pretendían fundarse en las experiencias similares de la primera mitad del siglo, con las que efectivamente había puntos de contacto -por ejemplo los discípulos de los cientistas sociales alemanes de los años 30-, lo cierto es que la concentración del esfuerzo en las nuevas escuelas de Sociología que organizan entre los años 70 y 80 es la que proporcionará las condiciones para la continuidad.

Enfocados en los asuntos prácticos, gran parte de quienes estudian la política panameña se han planteado los temas comunes de América Latina: las causas de la pobreza y el subdesarrollo, la dependencia, la deuda externa, el imperialismo político y financiero. Eso sí, la ciencia política panameña ha presentado una preocupación pronunciada por las relaciones internacionales, que 
es probable que no pueda enfatizarse suficientemente en este escrito, y que presenta múltiples facetas: jurídica, militar, económica, estratégica, ideológica. Por supuesto, el eje principal de esas reflexiones ha sido la relación con los Estados Unidos. La presencia de los Estados Unidos en la Zona del Canal, la pretensión panameña de verla terminada, la lucha por negociar unos nuevos Tratados que dispusieran la recuperación de ese territorio, pasando por el trauma de la invasión militar de 1989, la amenaza de establecer una nueva presencia militar estadounidense con el argumento de la lucha contra la droga, a mediados de los noventa, y seguidamente el reclamo en favor de la limpieza de las áreas dejadas contaminadas, y el tema de hoy: el Tratado de Libre Comercio con los Estados Unidos. La relación con los Estados Unidos es un asunto, además, relacionado con toda la idea de Nación Panameña, lo que explica la producción de interpretaciones de la separación de Panamá de Colombia -y reinterpretaciones, como se ha visto en los agrios debates suscitados en ocasión del centenario de la independencia-, sensibles tanto a la realidad histórica como a las necesidades del Estado y de la sociedad panameña.

Otro de los notables puntos de divergencia ha sido la caracterización del régimen militar panameño y la medida de su autoritarismo. Desde las perspectivas de muchos cientistas sociales, el valor de la democracia electoral era relativo, dado que serviría tan sólo para gestionar, aunque de otra forma, una sociedad esencialmente clasista. Ciertamente, los procesos electorales han sido revalorados en la teoría marxista de la política, y encontramos un renovado y generalizado interés por su profundización y ampliación, sin que esto signifique reconocer que la democracia se agota en las elecciones periódicas.

Respecto a estos asuntos, es evidente que la desaparición del régimen militar, la posterior devolución del Canal de Panamá (y el cierre de las bases militares estadounidenses), así como el fin de la Guerra Fría y la caída del muro de Berlín, han disipado en gran medida algunas de las más importantes causas de tensión en los estudios políticos panameños, lo que ha permitido y permitirá colaboraciones impensables hasta ahora. El potencial de la ciencia política en Panamá es así mayor que su impacto actual.

Sin embargo, hitos como la publicación de la Revista Tareas a lo largo de los años hablan de la demanda de información y de análisis de la realidad nacional, que superen la prensa de opinión. Mientras tanto es imperceptible la distinción entre el político y el politólogo. La valoración de uno y el otro corren la misma suerte, salvo que intervengan largas explicaciones.

Para concluir, hay que decir que el presente permite también el optimismo. Panamá envió una delegación al Primer Congreso Centroamericano de Ciencia Política (San José de Costa Rica, 2003), en el que presentó dos ponencias (Castillero Hoyos, 2003; y Sanmartín, 2003). En ese Congreso se gestó la continuidad de los esfuerzos regionales a favor de la disciplina, se fijaron las sedes de los siguientes Congresos Centroamericanos, su periodicidad (cada dos años), y se decidió la posterior creación de la Asociación Centroamericana de Ciencia Política²3.

En seguimiento de lo acordado en San José, en 2004 el CIDEM realizó en Panamá el Primer Encuentro Nacional de Politólogos, e instaló una Comisión Permanente de Ciencia Política, con el objeto de promover el avance de la disciplina. Actualmente se prepara la representación panameña al Segundo

23 A cargo de Giulia De Sanctis de la Universidad de Panamá, y Salvador Sánchez G., de la Universidad Católica Santa María La Antigua. 
Congreso Centroamericano, que tendrá lugar en Guatemala, en 2005. Sin exagerar el significado de estos eventos, puede decirse legítimamente que la ciencia política en Panamá tiene un nuevo punto de partida. Esperemos que el impulso hacia la institucionalización de la disciplina sea vigoroso y duradero.

\section{REFERENCIAS}

Adames Mayorga, Enoch. 2002. "Repensar las ciencias sociales: una perspectiva desde los sistemas-mundo". Tareas 112: 19-37. Adames Mayorga, Enoch. 2000. "Matriz Político-Institucional de la Gobernabilidad en Panamá". Tareas 106: 63-90.

Adames Mayorga, Enoch. 1998. "Partidos Políticos, proceso electoral y gobernabilidad democrática en Panamá: las elecciones de 1994". En Urnas y Desencanto Político. Elecciones y Democracia en América Latina 1992-1996, editado por Rial, Juan y Daniel Zovatto. San José de Costa Rica: IIDH-CAPEL, 225-263.

Adames Mayorga, Enoch. 1996. "La Relación Sociedad Civil-Estado en Hegel como Problema Histórico-Político de la Modernidad". Tareas 93: 103-118.

Aparicio, Fernando. 2003. "En defensa del 3 de noviembre". Revista Cultural Lotería 450-451: 14-33.

Arosemena, Justo. 1888. Estudios Constitucionales. Paris: A Roger y F. Chernoviz Editores.

Arosemena, Justo. 1979. "Apuntamientos para la Introducción a las Ciencias Morales y Política". En Patria y Federación, editado por Nils Castro. La Habana: Casa de las Américas, 71-213.

Arroyo Camacho, Dulio. 1980. El sistema de gobierno existente en Panamá luego de las últimas reformas a la Constitución nacional. Panamá: Ediciones Librería Cultural Panameña.

Arias Calderón, Ricardo. 2004. "Política y Gobernantes: Establecimiento de la República (1903-1931)". En Dimensiones de la Historia de Panamá, editado por Club Unión de Panamá. Panamá: Club Unión, 223-242.

Arias Calderón, Ricardo. 2001. Democracia sin Ejército. La Experiencia de Panamá. San José de Costa Rica: Fundación Arias para la Paz y el Progreso Humano.

Bernal V., Miguel Antonio. 2004. “Evolución constitucional desde la separación de Panamá”. En Historia General de Panamá (Volumen III, Tomo I), editado por Alfredo Castillero Calvo. Bogotá: Digital Designs Group, 34-48.

Bernal V., Miguel Antonio. 2000. Constituyente y democracia. Panamá: Editorial Chen, Instituto de Estudios Políticos e Internacionales.

Bernal V., Miguel Antonio. 1998 (1986). Militarismo y Administración de Justicia. Panamá: Editorial Portobelo.

Beluche, Olmedo. 2003. La verdadera historia de la separación de 1903: reflexiones en torno al centenario. Panamá: Imprenta Articsa.

Beluche, Olmedo. 1999. Estado, nación y clases sociales en Panamá. Panamá: Editorial Portobelo.

Beluche, Olmedo. 1991. "Los trabajadores del Estado y la teoría marxista de las clases sociales". Tareas 78: 113-144.

Brown Araúz, Harry. 2002. "Hacia la Consolidación del Sistema de Partidos Políticos Panameños (1990-1999)". Tareas 111: 5-26

Castillero Hoyos, Alfredo. 2003. "Problemas de la Democracia y la democratización en el Panamá bajo dominio colombiano (1853-1903)". Conferencia en el Primer Congreso Centroamericano de Ciencias Políticas, San José, Costa Rica.

Castillero Hoyos, Alfredo. 1996. Problems of Democracy and Democratization in Panama 1510-1989. Inglaterra: Tesis doctoral, Universidad de Portsmouth.

Castillero Hoyos, Alfredo. 1992. "On authoritarianism and democracy in Spanish America: An Examination of Cultural Determinism". Tesina de maestría en estudios políticos, Universidad de Warwick.

Castro H., Guillermo. 2004. "Pro Mundi Beneficio. Elementos para una historia ambiental de Panamá Il". En Historia General de Panamá (Volumen III, Tomo I), editada por Alfredo Castillero Calvo. Bogotá: Digital Designs Group, 130-151.

Castro H., Guillermo. 1991. "Panamá 1970-1990. Transitismo, Nación y Democracia”. En La Democracia en América Latina. Actualidad y Perspectivas, editado por Pablo González Casanova y Marcos Roitman Rosenmann (coordinadores). Universidad Complutense de Madrid.

Camargo, Juana. 1992. "Ocupación, crisis económica y educación”. Tareas 81: 59-63.

Conniff, Michael L. 1992. Panama and the United States: the Forced Alliance. Athens: The University of Georgia Press.

Cortéz, Dorindo 1986. F.F.A.A. y Poder Político en Panamá. Panamá:Talleres de Poligráfica. 
Davis Villalba, Enriqueta y Freddy Blanco Muñoz. 2001. Ciencia Política y Sociología Política (2 tomos). Panamá: Editorial Universitaria.

De Sanctis, Giulia. 1996. Sobre la Articulación Estado/Sociedad Civil en la "Introducción a la Crítica de la Filosofía del Derecho de Hegel" de Karl Marx. XIV Congreso Científico Nacional- Universidad de Panamá.

Escarreola Palacios, Rommel. 2002. "Consideraciones históricas sobre los acontecimientos del 3 de noviembre de 1903". Revista Parlamentaria Debate 3: 165-173.

Fábrega P., Jorge y Ricaurte Soler M. 2003. Bibliografía Jurídica de Panamá. Panamá: Editorial Álvarez.

Figueroa Navarro, Alfredo. 2004. "La Sociología en Panamá en el siglo XX”. En Panamá: Cien Años de República, editado por Comisión Universitaria del Centenario de la República. Panamá: Editorial Universitaria.

Figueroa Navarro, Alfredo. 1999. "Los cien primeros números de Tareas y su aporte a las Ciencias Sociales". Tareas 101: 127-136.

Figueroa Navarro, Alfredo. 1993. "Derrotero de las ciencias sociales en Panamá a fines del milenio". En Panamá, 90 años de República (Tomo I), editado por Presidencia de la República - Instituto Nacional de la Cultura. Panamá: Presidencia de la Republica- INAC.

Figueroa Navarro, Alfredo. 1991. "Sociología y Ciencias Sociales en el Panamá de los noventa". Revista Hombre y Cultura, Segunda Época, I (2). Panamá: Universidad de Panamá.

Figueroa Navarro, Alfredo. 1983. Estudio Introductorio: El Desarrollo de las Ciencias Sociales en Panamá. Panamá: Talleres de Dutigrafía, Biblioteca de la Cultura Panameña (Tomo 5).

Figueroa Navarro, Alfredo. 1978. Dominio y sociedad en el Panamá colombiano: 1821-1903. Panamá: Litho Imprenta Panamá. Franco Muñoz, Hernando. 1993. La Democracia Tutelada. Panamá: Talleres de Poligráfica.

Franco R., Bolívar E. 2001. Panamá: Los Partidos Políticos en los 90 entre elecciones y transformaciones. Panamá: Universidad de Panamá-Tribunal Electoral.

García Diez, Fátima. 2003. "Panamá”. En Partidos Políticos de América Latina. Centroamérica, México y República Dominicana, editado por Manuel Alcántara y Flavio Freindenberg. México: Fondo de Cultura Económica e Instituto Federal Electoral, 527-614.

Gandásegui h., Marco A. 2004. "Democracia y Movimientos Sociales en Panamá". En Panamá: Cien Años de República, editado por Comisión Universitaria del Centenario de la República. Panamá: Imprenta Universitaria.

Gandásegui h., Marco A. 1998. "Ciencia y Tecnología en el Sector de las Ciencias Sociales: Panamá". Tareas 98: 97-106.

Gandásegui h., Marco A. 1989. La Democracia en Panamá. México: Editorial Mestiza. Universidad Autónoma Metropolitana.

García, Olmedo. 2005. "La pobreza en el marco de la globalización en Panamá". Cuadernos Nacionales (3ra. Época) 4. Panamá: Instituto de Estudios Nacionales, 39-47.

González H., Simeón. 2001. Los dilemas de la transición: constituyente y democracia. Panamá: Instituto de Estudios Políticos e Internacionales.

González H., Simeón. 1995. Ciencia Política y Modernidad. Panamá: Editorial Portobelo.

González H., Simeón. 1994. Panamá 1968-1990. Ensayos de Sociología Política. Panamá: Imprenta Universitaria.

González H., Simeón. 1985. La Crisis del Torrijismo y las Elecciones de 1984. Panamá: Ediciones Horizonte.

González H., Simeón. 1983. Política y Derecho: Un análisis crítico. Panamá: Editorial Nuevo Siglo.

González Marcos, Miguel y Alfredo Castillero Hoyos. 2005. "At the Heart of the Planetary Democracy: The UN Democracy Caucus". En volumen preparado por la Fundación Heinrich Boll. Alemania: En imprenta.

González Marcos, Miguel. 1987. "La Democracia de Partidos en 1983: Modernización y desengaño". En Estudios de Derecho Constitucional Panameño, editado por Jorge Fábrega P. Panamá: Editora Jurídica Panameña.

Guevara Mann, Carlos. 2004. "Calidad de la Representación Política y tamaño de las circunscripciones electorales: una comparación de las asambleas panameñas de 1945 y 1999". Revista Ciencia Política XXIV (2): 94-115.

Guevara Mann, Carlos. 2004a. "Auge y militarización de los organismos de seguridad pública: 1930-1990". En Historia General de Panamá (Volumen III, Tomo II), editado por Alfredo Castillero Calvo. Bogotá: Digital Designs Group, 63-74.

Guevara Mann, Carlos. 2000. "Patronage Distribution, Party Switching, and Electoral Manipulation by Panamanian Legislators: The Electoral Connection". Presentación en la reunión de la Asociación de Estudios Latinoamericanos (LASA), Miami. 
Guevara Mann, Carlos. 1999. C.M.A.: Reelección e Institucionalidad Democrática. Panamá: Instituto de Estudios Políticos e Internacionales y Editorial Portobelo.

Guevara Mann, Carlos. 1994. Ilegitimidad y Hegemonía. Una interpretación Histórica del Militarismo Panameño. Panamá: Editorial La Prensa.

Janson Pérez, Brittmarie. 1997. Golpes y Tratados: piezas para el rompecabezas de nuestra historia. Panamá: Instituto de Estudios Políticos e Internacionales.

Janson Pérez, Brittmarie. 1994. Panamá Protesta. 1968-1989. Panamá: Instituto de Estudios Políticos e Internacionales. Janson Pérez, Brittmarie. 1993. Panamá Protesta. 1968-1989. Panamá: Editorial La Prensa.

Lasso, Marixa A. 2004. "La crisis política post-independentista: 1821-1841". En Historia General de Panamá (Volumen II), editado por Alfredo Castillero Calvo. Bogotá: Digital Designs Group, 63-76.

Leis, Raúl Alberto. 2004. "Panamá: centralismo y metropolitización”. En Panamá: Cien Años de República, editado por Comisión Universitaria del Centenario de la República. Panamá: Editorial Universitaria.

Leis, Raúl Alberto. 2000. Abrir canales de participación: descentralización y poder local. Panamá: CONADEL.

Leis, Raúl Alberto. 1984. Radiografía de los Partidos. Panamá: Ediciones Centro de Capacitación Social.

McKay, Alberto. 2004. "Las Primeras crisis políticas". En Historia General de Panamá (Volumen III, Tomo II), editado por Alfredo Castillero Calvo. Bogotá: Digital Designs Group, 3-24.

Maloney, Gerardo. 2004. "Significado de la presencia y contribución del afropanameño a la nación panameña". En Historia General de Panamá (Volumen III, Tomo I), editado por Alfredo Castillero Calvo. Bogotá: Digital Designs Group, 152-171.

Millet, Richard L. 1996. "Panama: transactional democracy." En Constructing democratic governance: Mexico, Central America and the Caribbean in the 1990's, editado por Jorge Domínguez y Abraham F. Lowenthal. Baltimore y Londres: Johns Hopkins University Press, 92-104.

Moscote, José Dolores. 1979 (1929). "Relaciones de la Ciencia Política y del Derecho Constitucional con las Demás Ciencias". Revista Lotería 23: 278-279.

Noel, Enrique. 1999. "Democracia y Crisis Política”. Tareas 102: 117-132.

Noriega de Jurado, Jilma. 1978. Verdad y Miseria de nuestros partidos políticos. Panamá: Editora Renovación.

Ortega Durán, Oydén. 1989. Curso de Instituciones Políticas. Panamá: Imprenta de la Universidad de Panamá.

Ortega Brandao, Ismael. 1931. La jornada del día 3 de noviembre de 1903 y sus antecedentes. Panamá: Imprenta Nacional.

Otero, Patricia. 2002. Partidos y Sistemas de Partidos en Panamá: Un estudio de la Estructuración Ideológica. X Encuentro de Latinoamericanistas Españoles, Salamanca.

Pedreschi, Carlos Bolívar. 2004. "La Dictadura Militar". En Historia General de Panamá (Volumen III, Tomo II), editado por Alfredo Castillero Calvo. Bogotá: Digital Designs Group, 75-92.

Pedreschi, Carlos Bolívar. 1986. "El Dr. José Dolores Moscote y las Formas Ideológicas del Estado Panameño". Iustitia et Puchritudo 4: 11-16.

Pereira Burgos, César. 1991. La democracia cuartelera. San José de Costa Rica: Editora Dos Mares.

Pereira Burgos, César. 1986. Libertad y Autoridad. Panamá: Editora Dos Mares.

Pereira, Renato. 1978. Fragmento de Fuerzas Armadas y Política, Panamá: Ediciones Nueva Universidad, Imprenta Universitaria.

Pérez, Orlando J. 1995. "Elections under crisis: background to Panama in the 1980's". En Elections and democracy in Central America, editado por John Booth y Mitchel Seligson. Carolina del Norte: The University of North Carolina Press, 123-151.

Pérez, Orlando J. 1993. "Tendencias de Investigación sobre los Sistemas Electorales y su Impacto en el Sistema Político". Tareas 85: 65-81.

Pinzón, Milcíades. 1988. "La crisis de los ochenta en Panamá". Tareas 68: 25-51.

Pippin, Larry LaRae. 1964. The Remon Era: an Analysis of a Decade of Events in Panama, 1947-1957. Standford: Institute of Hispanic American and Luso-Brazilian Studies.

Porcell G., Nestor. "Apuntes sobre la Sociología Marxista". Tareas 13: 5-14.

Porcell G., Néstor. "La metodología general de las ciencias sociales". Revista Lotería 376: 34-46.

Porras, Hernán. 1953. "Papel Histórico de los grupos humanos en Panamá”. En Panamá: 50 años de República, editado por Junta Nacional del Cincuentenario. Panamá: Ediciones de la Junta Nacional del Cincuentenario, 63-107. 
Quintero Correa, César. 1973. Principios de Ciencia Política. Panamá: Ediciones de la Librería Cultural Panameña.

Ricord, Humberto E. 2003. El 3 de noviembre de 1903, visto desde el Centenario. La separación de Colombia. 2t., Panamá: Editora Sibauste.

Ricord, Humberto E. 1991. Panamá y Noriega: Orgía y aplastamiento de la narcodictadura. México: Impresora Eficiencia.

Ricord, Humberto E. 1990. "Presidencia, Parlamento y Dictadura Militar en Panamá". Anuario de Derecho XVIII (19): 141-161.

Ricord, Humberto E. 1983. Los clanes de la Oligarquía y el golpe militar de 1968. (s.l.): (s.n.).

Ritter Guevara, Vilma. 1983. "Esbozo teórico sobre las intervenciones militares en América Latina. Teoría del Golpe Militar". En

El Desarrollo de las Ciencias Sociales en Panamá (Tomo 5), editado por Alfredo Figueroa Navarro. Panamá: Biblioteca de la Cultura Panameña.

Ritter Guevara, Vilma. 1977. L'Argentine, L'Armée et le Péronisme. Mémoire DES, Sciences Politiques, Université de Paris-I, París.

Ritter Guevara, Vilma.1977a. Les Relations Civils-Militaires au Brésil (1886-1954; le Getulismo (1930-1954), Thése de $3^{e}$ cycle, Sciences Politiques, Université de Paris I (Panthéon-Sorbonne), Paris.

Rodríguez Patiño, Rubén Darío. 2005. Ciencia Política, Temas y Programas. Panamá: Editorial Portobelo.

Rodríguez Patiño, Rubén Darío. 2004. Encuentros y Desencuentros. Ciencia Política y Ciencias Políticas. Panamá: Editorial Portobelo.

Rodríguez Patiño, Rubén Darío. 1997. "De la Democracia Censitaria a la Democracia Participativa". Anuario de la Facultad de Derecho y Ciencias Políticas XXII (25-26): 10-21.

Rodríguez Patiño, Rubén Darío. 1998. El Príncipe de Nicolás Maquiavelo ¿Ciencia o Técnica de la Política? Panamá: Editorial Portobelo.

Rodríguez Patiño, Rubén Darío. 1998. El Concepto de Política. Panamá: Editorial Portobelo.

Ropp, Steve C. 1982. Panamanian Politics: From Guarded Nation to National Guard. New York: Praeger.

Sánchez, Peter M. 2000. "Consolidating Democracy in Panama. Problems and Prospects". Presentación en el Congreso de la Asociación de Estudios Latinoamericanos (LASA), Miami.

Sánchez González, Salvador. 2004. "Cien años de Labor Legislativa”. En Historia General de Panamá (Volumen III, Tomo I), editado por Alfredo Castillero Calvo. Bogotá: Digital Designs Group, 355-372.

Sánchez González, Salvador. 2004ª "La Transición a la Democracia en Panamá". En Historia General de Panamá (Volumen III, Tomo II), editado por Alfredo Castillero Calvo. Bogotá: Digital Designs Group, 283-301.

Sánchez González, Salvador. 2003. "Informe sobre Panamá". En Democratización Interna de Partidos Políticos en Centroamérica: Avances y Tareas Pendientes, editado por José Thompson. San José de Costa Rica: IIDH-Capel, 379-458.

Sánchez González, Salvador. 1999. "Transición a la Democracia y El Ocaso de los Militares”. En Transiciones a la Democracia en Centroamérica, editado por Jorge Mario García La Guardia. San José de Costa Rica: FUNPADEM, 184-190.

Sánchez González, Salvador. 1996. El Financiamiento de los Partidos Políticos en Panamá. Fundación para el Desarrollo de la Libertad Ciudadana. Panamá: Editora Libertaria (Ediliber).

Sánchez González, Salvador. 1995. La Desobediencia Civil en Panamá. Panamá: Editora Libertaria (Ediliber).

Sanmartín, Antonio. 2003. "Dualidad Socioeconómica y Partidos Políticos en América Latina”. Conferencia en el Primer Congreso Centroamericano de Ciencia Política. San José, Costa Rica.

Scranton, MargaretE. 1991. The Noriega Years: U.S.-Panamanian Relations, 1981-1990. Boulder: Lynne Rienner Publishers. Soler, Ricaurte. 1988. Estudio introductorio y antología: El pensamiento político en Panamá: Siglo XIX y XX. Panamá: Editorial Universitaria, Biblioteca Cultural Panameña.

Soler, Ricaurte. 1985. TAREAS: 25 años de Existencia. Tareas 60: 3-4.

Soler, Ricaurte. 1975. Panamá, Nación y Oligarquía. Panamá: Ediciones de la Revista Tareas.

Soler, Ricaurte. 1963. Formas Ideológicas de la Nación Panameña. Panamá: Ediciones de la Revista Tareas.

Soler, Ricaurte. 1954. Pensamiento panameño y concepción de la nacionalidad durante el siglo XIX: para la historia de las ideas en el Istmo. Panamá: Imprenta Nacional.

Soler Torrijos, Giancarlo. 1988. "Surgimiento del reformismo militar, evolución y crisis". En Panamá: fuerzas armadas y cuestión nacional. Panamá: Taller de Estudios Laborales y Sociales. 
Terán, Oscar. 1976. Del Tratado Herrán-Hay al Tratado Hay-Bunau Varilla. Historia crítica del atraco yanqui, mal llamado en Colombia la pérdida de Panamá y en Panamá nuestra independencia de Colombia. Bogotá: Valencia Editores.

Ungo, Urania. 1993. "Regresar a Hegel: El Fin de la Historia, según Francis Fukuyama". Tareas 85: 93-102.

Valdés Escoffery, Eduardo. 1999. La elección de los legisladores en circuitos plurinominales. Panamá: Tribunal Electoral.

Vega, Lina. 2004. "El Municipio, o Cien Años de Espera”. En Historia General de Panamá (Volumen III, Tomo I), editado por Alfredo Castillero Calvo. Digital Designs Group, 373-390.

Salvador Sánchez González es especialista en Derecho Constitucional y Ciencia Política por el Centro de Estudios Constitucionales de Madrid, España. Licenciado en Derecho y Ciencias Políticas por la Universidad Católica Santa María La Antigua (USMA), de Panamá, República de Panamá. Ha sido profesor universitario desde 1993 en la USMA, donde ha dictado los cursos de Introducción a la Ciencia Política, Historia de las Ideas Políticas, Derecho Constitucional, y Derechos Humanos. Entre sus publicaciones se encuentran: La Desobediencia Civil en Panamá (1995), Aproximación al Financiamiento de los Partidos Políticos en Panamá (1997), Introducción a la Defensoría del Pueblo (en conjunto con Gisela De León, 2001) y diversos artículos sobre Derechos Humanos, Derecho Constitucional y Parlamentario, así como sobre los partidos políticos, y la transición panameña a la democracia. También fue miembro fundador del Centro de Iniciativas Democráticas (CIDEM), del que fue su primer Presidente.

(E-mail:salvasan@pty.com) 